\title{
MRI/CT SCAN UNDER SEDATION IN CHILDREN
}

\author{
S. Amin ${ }^{1}$, H. Mazhar ${ }^{2}$, P. Heaton ${ }^{2}$ \\ ${ }^{1}$ Paediatric Department, ${ }^{2}$ Yeovil District Hospital, Yeovil, UK
}

Background: Children who need MR scanning frequently need sedation in order to remain still for the procedure. This poses a number of potential problems including the organizational difficulties in ensuring that the child is admitted, sedated and scanned all in a timely, coordinated and safe manner.

Methods: We conducted an audit/retrospective study to review our practice by examining our safety and success rates. The medical notes of 56 patients from June 2009-December 2010 were reviewed. We looked at the age, gender, date, indication, emergency/planned, oral Chloral Hydrate, IV midazolam, accompanied by $\mathrm{SHO} / \mathrm{SpR}$, success rate, complications, result, picture quality and whether any repeat scan was required.

Results: The majority of scans were performed as planned procedures. 54(98\%) patients received oral chloral hydrate $50-100 \mathrm{mg} / \mathrm{kg}$ and $44(82 \%)$ of them received IV midazolam $100 \mathrm{mcg} / \mathrm{kg}$. Only $12(28 \%)$ of the midazolam group required a further dose. $47(84 \%)$ had successful sedation with good quality pictures and only 4 required a repeat scan. We found that most patients were pre-school children, $(60 \%)$ were aged 15 years. Half of our patients were accompanied by SpRs during the procedure and no complications were reported. The commonest indication was for seizures and the majority of scan results showed incidental findings.

Conclusions: We had a high success rate when compared with published data and we considered whether this was due to the frequent use of midazolam or because Chloral Hydrate was used in conjunction with the midazolam. We believe implementation of this protocol in children is safe, successful and cost effective. 